\title{
Perception, Acceptance, and Hesitancy of the Public Regarding Covid-19 Vaccine and Immunization: A Literature Review
}

\author{
Hamdia Ahmed ${ }^{1}$, Karim Aziz ${ }^{2}$ and Ahmed $\mathrm{Ali}^{3, *}$ \\ ${ }^{1}$ Professor in Maternity of Nursing, Hawler Medical University/ College of Health Science \\ ${ }^{2}$ Assist Prof. Community health nursing, Hawler Medical University/ College of Nursing \\ ${ }^{3}$ Clinical instructor, Hawler Medical University/ College of Nursing \\ *Corresponding author. Email: ahmed.naifali87@gmail.com
}

\begin{abstract}
Background: Developing a vaccine against COVID-19 is widely regarded as a critical method for containing the pandemic. Public adoption, on the other hand, is contingent on one's view's to accept and perceptions about the vaccine. The aim of this literature review is to determine the factors that influence public acceptance and hesitancy against Covid-
\end{abstract} 19 vaccines.

Objective: To determine the sociodemographic features associated with the public's understanding and acceptance or fear of Covid-19 vaccines. To ascertain the causes that contribute to acceptance or refusal of the Covid vaccine.

Method: 22 articles were searched and selected through PubMed database, Google Scholar database, and Scopus database between January 1st, 2020 to 30th December 2020.

Discussion: The finding indicates that the United States and the United Kingdom have done the most research on the adoption and rejection of the Covid-19 vaccine, whereas China's experiments have the largest citation rate. The literature indicates that the Australian community has the highest acceptance rate, whereas the Netherlands has the highest distrust and hesitancy toward the Covid-19 vaccine.

Conclusion: The Covid-19 virus is urged to be given to the population's needs toward the vaccine but at the same time acceptance rate was varied between each countries population and fear of conspiracy ideas from some countries. Some countries are concerned about the safety of the vaccine being given to their citizens.

Keywords: vaccine acceptance, vaccine hesitancy, Covid-19 vaccine.

\section{INTRODUCTION}

severe acute respiratory syndrome coronavirus 2 (Sars-Cov-2), the virus that causes covid-19, reached pandemic status on March 11th, 2020. over 5.5 million individuals have been infected with the virus, which has resulted in over 350,000 fatalities. over the following two years, the worldwide economic impact of this epidemic is expected to amount to a nine trillion dollar loss in productivity. in the united states, the first human clinical study of vaccination began on march 3rd, 2020, with a predicted completion date of june 1st, 2020. the successful development, production, and distribution of any vaccine are, however, insufficient to guarantee adequate vaccine coverage [1].

vaccination has a low level of purpose, which may be understood in the idea of hesitancy. vaccine hesitancy could increase the risk of individuals acquiring the disease and can reveal a public health risk (greater virus transmission). even the best vaccines cannot prevent the 
disease if they are not used consistently. the vaccine rejection problem involves several factors, and in many nations, it affects a wide range of individuals. ending the current pandemic needs an understanding of the reasons widespread worries about the united states covid-19 vaccination and techniques for overcoming such hesitation. it is important to understand different vaccine attitudes because a heterogeneous approach to vaccine refusal that deals with different groups' concerns are more successful than a homogeneous strategy. given recent warnings that a vaccine might be distributed even before clinical trials are completed, a qualitative and quantitative assessment of attitudes toward vaccine development and confidence is extremely important in the united states. [2], [3].

Many covid-19 conspiracy theories emerged due to the pandemic. because vaccination beliefs tend to negatively affect people's intentions to vaccinate, this could become a crucial matter for the near future. conspiracy theories are reasons to understand the ultimate reasons for significant social and political events by citing one or more influential performers [4].

Although the total number of vaccines decreased the incidence of these vaccinations changed and some might even not receive vaccinations at all, so we must investigate how social media affects how people change their minds and their attitudes about vaccination. every province's economic turmoil raises population resentment against the scenario and refuses the vaccine. [5].

This study aims to explore the factors influencing acceptance, the hesitancy of the public toward covid-19 vaccines.

1. to determine the sociodemographic factors that influence the public's perception and acceptance of covid19 vaccinations.

2. to determine the factors that influence whether or not a person accepts or refuses the covid vaccination.

\section{MATERIAL AND METHOD}

\subsection{Data Source}

The articles were searched through PubMed database, google scholar database, and Scopus database by using boolean statement terms, of ("perception" and "attitude" and "public" or " acceptance" and " covid-19 vaccines" or "covid-19 vaccination") between January 1st, 2020 to 30th December 2020.

\subsection{Selection Criteria}

Inclusion criteria: after the search result from databases, the inclusion criteria were only English language articles, from medical journals and nursing journals, articles that meet the terms and objectives were included.

\subsection{Exclusion criteria}

articles of none English languages, not between January 1st, 2020 to 30th December 2020, objectives not meeting the study aims and objectives themes, and review articles, systematic review, meta-analysis studies, and journal conferences were excluded.

\subsection{Screening}

66 articles were searched from databases selected, after further screening from the title, abstract, and content of the studies only 22 articles were selected for the literature review due to different aims, objectives, unmatching the selection criteria from other articles.

\subsection{Studies included for the literature review}

\begin{tabular}{|c|c|c|c|}
\hline $\begin{array}{l}\text { Author } \\
\mathrm{s}\end{array}$ & Year & country & $\begin{array}{l}\text { sample } \\
\text { s size }\end{array}$ \\
\hline 1. [6] & $\begin{array}{l}202 \\
0\end{array}$ & China & 2058 \\
\hline 2. [7] & $\begin{array}{l}202 \\
0\end{array}$ & China & 352 \\
\hline 3. [1] & $\begin{array}{l}202 \\
0\end{array}$ & Ireland & 1,041 \\
\hline 4. [8] & $\begin{array}{l}202 \\
0\end{array}$ & Malta & 9681 \\
\hline 5. [9] & $\begin{array}{l}202 \\
0\end{array}$ & Malaysia & 1,159 \\
\hline 6. [10] & $\begin{array}{l}202 \\
0\end{array}$ & $\begin{array}{l}\text { United } \\
\text { Kingdom }\end{array}$ & 527 \\
\hline 7. [11] & $\begin{array}{l}202 \\
0\end{array}$ & Italy & 1004 \\
\hline 8. [12] & $\begin{array}{l}202 \\
0\end{array}$ & Nigeria & 465 \\
\hline 9. [2] & $\begin{array}{l}202 \\
0\end{array}$ & $\begin{array}{l}\text { United } \\
\text { States of } \\
\text { America }\end{array}$ & 316 \\
\hline 10. [5] & $\begin{array}{l}202 \\
0\end{array}$ & France & 5018 \\
\hline 11. [4] & $\begin{array}{l}202 \\
0\end{array}$ & France & 778 \\
\hline 12. [13] & $\begin{array}{l}202 \\
0\end{array}$ & $\begin{array}{l}\text { United } \\
\text { Kingdom }\end{array}$ & 5,114 \\
\hline 13. [14] & $\begin{array}{l}202 \\
0\end{array}$ & $\begin{array}{l}\text { United } \\
\text { States of } \\
\text { America }\end{array}$ & 1062 \\
\hline 14. [3] & $\begin{array}{l}202 \\
0\end{array}$ & Israel & 398 \\
\hline
\end{tabular}




$\begin{array}{llll}\text { 15. [15] } & 202 & \text { Saudi } & 3,101 \\ & 0 & \text { Arabia } & \\ \text { 16. [16] } & 202 & \text { Netherlan } & 698 \\ & 0 & \text { d } & \\ \text { 17. [17] } & 202 & \text { United } & 534 \\ & 0 & \begin{array}{l}\text { States of } \\ \text { America }\end{array} & \\ & & \text { Amdia } & 351 \\ \text { 18. [18] } & 202 & \text { India } \\ & 0 & & \\ \text { 19. [19] } & 202 & \text { United } & 32,361 \\ & 0 & \text { Kingdom } & \\ \text { 20. [20] } & 202 & \text { Nigeria } & 517 \\ & 0 & & \\ \text { 21. [21] } & 202 & \text { Australia } & 1143 \\ & 0 & & \end{array}$

\section{FINDING}

\subsection{Sociodemographic characteristics of the public regarding perception, acceptance, attitude toward Covid-19 vaccines}

The distribution of members in the community showed that being male, and married, having a high risk of infection, having been vaccinated in the previous season, and believing in the Covid-19 vaccine's effectiveness, and valuing doctor's advice could impede immediate vaccination. [6].

Age, gender, educational level, religion, employment, monthly income, and geographical zone had little effect on respondents' perceptions of and preparedness to engage in the COVID-19. [12]. Young age, female gender, lower-class, and racial and ethnic identity were all linked to hesitation. With the minimal change, socioeconomic ideas were clarified. [13]. females were more likely to agree with the statement of accepting than males. The older the person is, the more likely they are to agree with the statement than a younger person [21]. Whereas in this study it will be on the contrary side that men and educated males and others who got the seasonal influenza vaccine are most prone to catch the flu. The previous year's declaration suggested a stronger desire to obtain Covid-19 vaccination [3].

To anyone that does not have faith in vaccines, they would appear to be fewer with vaccinations. It is necessary to provide specifically focused education messaging for college students to emphasize the danger of the COVID-19 illness., suggest educators, so the education level will also have an important effect on the understanding vaccination and immunization to accept the idea of it and perceiving side effects from the vaccine itself [14]
As this study reveals that occupational differences enhance and provide an easy environment such males were more willing to consent to the vaccination. The specialty of medical doctors fulfilled the highest expected vaccines take. There have been fears that people considering vaccines can't truly understand what are the vaccines, what is their risk and on the other side, they can provide information to help society to release their stress about it [8].

\subsection{Factors associated with accepting or refusing Covid-19 vaccine}

There are many factors affecting people perception, attitude, acceptability toward Covid-19 vaccines and all other vaccines inoculated from the time being, even more from demographic factors such as age, sex, educational level, economic status, and occupational differences, whereas interpersonal factors such as fear of new vaccines, hesitancy, conspiracy, individuals point of view regarding politics and overall aspects of life [7], [6], [4], [20].

\subsection{Perception and attitude regarding Covid-19 vaccine taking}

According to this study, The majority of the participants got their knowledge about Covid-19 through social media. while nearly had a poor perception of the trial. Age, gender, educational level, religion, occupation, monthly income, and geographical zone did not affect participants' willingness to engage. [12].

Most of the participants have a confident perception of the regulated vaccine and they can take the vaccine unless the dangers of vaccines outweigh the advantages in connection with the wellbeing and medical conditions [2]. Perceived seriousness of the Covid-19 and fear of the Covid-19 vaccination were linked with higher vaccine uptake. Many facets of this condition are still unclear and its medical requirements are still not well established. Reports of the perceived seriousness may affect the propensity to get a vaccination against covid-19 in particular age groups [14].

The perception of most of the respondents about the Covid-19 vaccine was positively able to take the COVID19 vaccine, but a few were reluctant. Our results indicate that vaccination is important to warn the public that the vaccine will be secure and it will function. Besides, there is a need to make sure that the public trust in the vaccine is won and that both communities are involved adequately to ensure a favorable reaction to the vaccine [12], [19].

The study found negative attitudes toward general vaccines as mentioned possible side effects and long-term damage did not play a minor role in people's hesitancy to receiving vaccinations. The key strength point is to shift the attitude of the members of the public toward 
vaccination and to increase covid-19 vaccine take-up [14].

This study noticed that two variables greatly affect the respondents' attitudes and beliefs regarding a COVID-19 vaccine. We find important influences of the timing, potency, and place of vaccine administration on vaccine uptake. Attempts to resolve numerous issues, particularly vaccination formulation, evaluation of vaccinations, and community engagement efforts, are expected to improve vaccination use and better prevent the COVID disease outbreak [2].

Distrustful attitudes towards vaccination were higher amongst individuals from ethnic minority backgrounds. A small number of people said they didn't want to have the COVID-19 vaccination. while the majority were unsure. Low-income groups and those who had not got a flu vaccination the previous year were the strongest predictors of vaccine doubt and rejection. Female gender, having children, not following government rules, and having a low income were all influences. [20]. This research presents an understanding of public perception about a potential 50 COVID-19 vaccine and indicates a basic framework for understanding vaccine attitudes. To launch the latest vaccinations, governments need to consider the needs of both the populations and the policies that can facilitate successful dialogue with those communities [21].

\subsection{Hesitancy and fear among the public toward Covid-19 vaccine}

The hesitation to the covid-19 vaccination is evenly spread among the population.. There is a strong correlation between readiness to take a vaccination and awareness of the mutual value. Public awareness encouraging the prosocial effects of vaccines can be more efficient. Beliefs that encourage distrust and decrease social stability can result in lower vaccination rates [13].

While as its mentioned in this study various questions raised by the participants about the COVID-19 trial's findings are presented as a plurality. are fearful of the potential adverse effects and complication of these experiments. the vaccination, minorities are fearful of it being used as a chemical agent while the majorities are afraid of vaccine-related mortality [12].

Hesitancy and risk of taking the COVID-19 vaccine are linked to whether someone has already been vaccinated for influenza. Concerns were posed concerning the absence of knowledge about the novel vaccination, particularly about not knowing the long-term consequences of the vaccine especially hesitancy of having a serious side effect from the vaccine [8].

This study demonstrated that in Ireland and the United Kingdom, vaccine-hesitant/resistant participants varied concerning a variety of sociodemographic, diplomatic, and Variables related to wellbeing, but across a broader spectrum of psychological structures, were comparable. Those who were immune to the covid-19 vaccine in both populations were less able to receive information from conventional and reliable sources about the pandemic and had comparable data. In these sources, degrees of distrust. The current results can enable public health officials to approach vaccine-hesitant and resistant individuals more effectively, establish appropriate communication techniques that take into account their unique psychological arrangements and exploit networks of distribution that can reach these individuals successfully [1].

As already stated More than half of the hesitant participants expressed fear about potential adverse effects as one of the most prevalent reasons for their apprehension., they think that covid-19 vaccines are not safe due to multiple factors from health promotion programs to the individual educational programs to nourish people about the coronavirus vaccines and As a result, it is insufficient for an effective coronavirus immunization campaign. When we compare our findings to those of comparable research conducted in the United Kingdom and Ireland, we find that acceptance rates are lower and hesitation rates are greater. [16].

The fear of the Covid-19 vaccine has been mentioned in this study [15] as participants questioned about the safety of the vaccine, the majority of them were afraid of the vaccine and not sure about the decision making to take it or not due to side effects, the biomedical advertisement from the media and social network even though the complication questionnaires drown attention of the participant to be afraid about the mortality rate and consequences of the Covid-19 vaccine.

\subsection{Conspiracy belief among population toward Covid-19 vaccine}

There were three primary reasons for rejecting the coronavirus vaccine. According to the explanation for rejecting the vaccine, the logistic simulations did not find any in several consequences of political approach and political conspiracy. the belief that a vaccine developed in a hurry is too risky was one of three major factors. Men who were more likely to reject the vaccine than women were often considered useless because of the harmless presence of COVID-19 dominant classes and Women were more likely than males to reject the vaccination. Besides, the vaccine is likely to be against the health concept and conspiracy ideology fortified populations attention against the vaccine [5]

Conspiracy theories arose in tandem with the COVID19 epidemic. The researchers focused on the connection between conspiracy, vaccination views, and vaccine intention. The study found that conspiracy beliefs about chloroquine negatively predicted participants' intentions. 
Implications for the pandemic and potential responses are discussed, and we also observed that COVID-19 The strategy for future vaccination of participants against COVID-19 was adversely forecast for conspiracy convictions (including chloroquine conspiracy ideas) and conspiracies. Finally, conspiracy suspicions projected approval for chloroquine as a COVID drug Interestingly, the risks of the vaccinations were not pointed to by any of the conspiracy beliefs. Consequences for the pandemic and future reactions are presented [4].

In the case of COVID vaccination adverse effects, the majority of the study population was worried. The minority of responders lacked trust in the efficacy of human and human vaccination and conspiracy, whereas the political conspiracy fulfilled the population's mind and brain. A large number of people thought that they had other reasons to avoid being vaccinated [18].

Almost half of the participants had hesitancy about Covid-19 vaccines, the variables were correlated with younger age, female sex, high poverty, and race, and the majority were willing to be vaccinated. An individual's ability to receive a vaccine relies on how well they understand vaccinations add favorably to our lives. Some issues will significantly hinder vaccination adoption, such as those concerning conspiracies and distrust of physicians. We should disseminate knowledge regarding the prosocial effects of the vaccination to reassure the public [13]. This study [17] also demonstrates that Individual variations in vaccination intentions show causes and targets that could help measures to encourage vaccination, such as preference strategies and bundling vaccine rates in vaccine taking actions or belief in conspiracy theories.

\subsection{The willingness of people to take the Covid-19 vaccine}

Concerning the production of the COVID-19 vaccine, most health care workers believed it was significant. The HCWs noticed a greater tolerance for the potential vaccine. Around half of the health care staff assumed that vaccines developed domestically would be safer than those produced overseas, lower than the general public [7]. During the 2019 pandemic, the review investigated at the acceptability of the COVID-19 vaccine among Chinese people. More than half of survey participants decided to receive the vaccine as soon as it became available. The participants were primarily interested in obtaining Covid-19 as soon as its production and approval process was completed successfully. Most people accepted that both the immunization plans and both forms of vaccines were safe [6].

On the contrary, reveals that misinformation and propaganda contribute to vaccine denial and vaccine refusal. This threatens attempts to eliminate the coronavirus outbreak. Public health workers need to recognize the importance of safe and potent vaccines in combating this novel and deadly virus [12]. Individuals who were distrustful of vaccination appeared to come from minority communities. Approximately half of the respondents said they would refuse to have a COVID-19 vaccination, and lower levels of vaccine bewilderment and rejection are associated with low-income groups and those who did not get a flu vaccine the previous year. [20].

A majority of the research population decided that they would consent to receive vaccines if they have been proved safe and reliable. many of the respondents believe that vaccines should be performed if a physician recommends them. Fewer than half of the sample decided to get vaccinated even though it was offered free of charge [15], [18].

While on the opposite side the price of the vaccine was not found to substantially influence the probability of vaccination, and the gender of the participants was the only demographic factor affecting intention to take the vaccine as it showed in this study [9].

Currently, acceptance of the COVID-19 vaccine is high. high-risk populations. Media strategies need to use social media and knowledge about health, emotional, social, and environmental effects, and salience of consequences [10].

\section{FIGURE}

\subsection{Acceptance range of Covid-19 vaccines among different countries}

Figure 1. acceptance of Covid-19 vaccines per different countries

st

COVID-19 VA C CINE ACCEPTANCE\%

90

8078

70

60

50

40

30

20

10

0

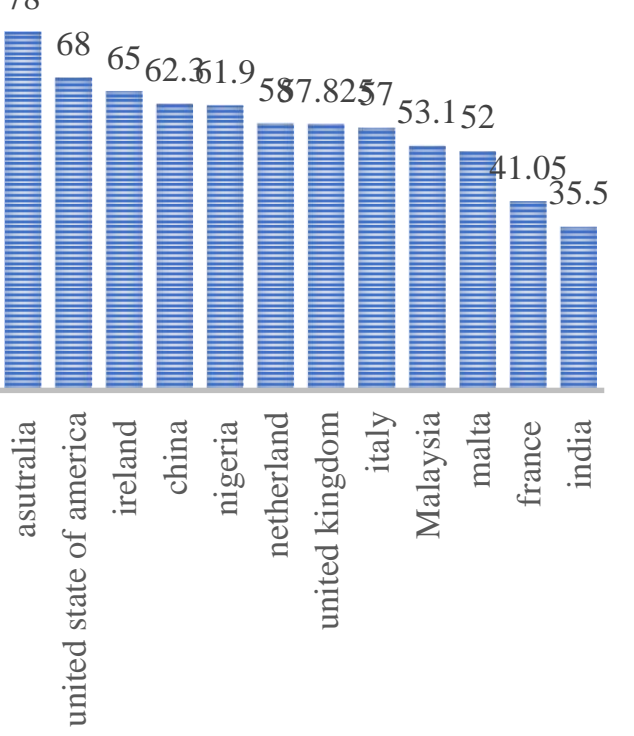


Figure 1 demonstrates that acceptance of covid-19 vaccines has a higher percentage among the Australian population which is $78 \%$ of them accepting the vaccine when it is available as mentioned in this study [21], this will insures that the demand and public understanding of covid-19 vaccines raise their attention and seriousness of coronavirus infection. while the united states this study [2], [17] show $68 \%$ acceptance and willingness to take a vaccine, on the other side united kingdom has shown 57\% of acceptance among the population due to fear and hesitancy of the public from the side effect of the vaccines and seriousness of its complication as it's proven in this study [10], [19], lastly this study [18] in India shows lower acceptance of $35 \%$ due to public misunderstanding about covid-19 virus, idea that herd immunity will develop after recovery, and a group of them will accept it if the vaccine free of charge.

\subsection{Fear, and hesitancy of Covid-19 vaccines among the population}

Figure 2. No table of figures entries found.: fear and hesitancy of Covid-19 vaccines per country

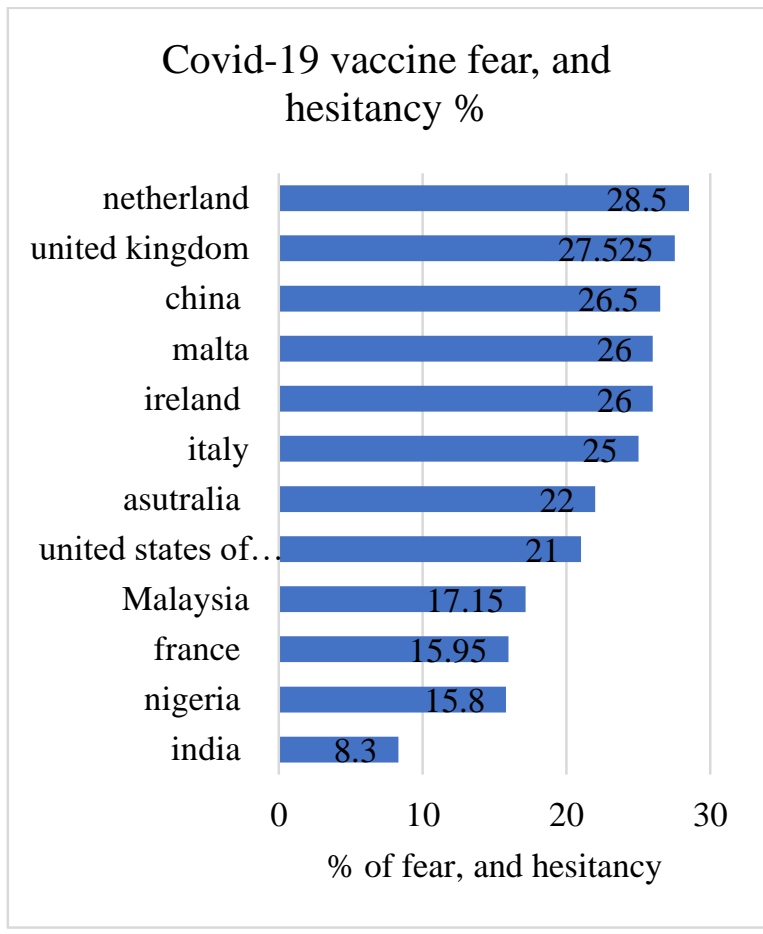

Figure 2 demonstrates that Netherland has a higher percentage of fear and hesitancy toward Covid-19 vaccines as a result of multiple factors shown in the study [16] such as conspiracy of the Covid-19 virus, political conspiracy, and even hesitancy from the side effect of the vaccine itself. On the contrary side, India has shown the lowest fear and resistance against Covid-19 vaccine taking as it's mentioned in this study [18]. While united states of America's population shows moderate fear and negative attitude toward the Covid-19 vaccine, however, the fear, negative perception and attitude can be controlled by health promotion programs such as doctors recommendation, governmental regulation for the vaccine administration as its mentioned in this studies [2], [17]. The conspiracy belief and ideology among the people of Italy made them overwhelming and afraid to accept the vaccine as demonstrated from this study [14], almost half of them will refuse the Covid-19 vaccines due to solid negative perception that the vaccines have certainty of a political conspiracy.

\section{DISCUSSION}

According to this study, the majority of individuals got their covid-19 knowledge through social media. Age, gender, educational level, religion, occupation, monthly income, and geographical zone did not affect participants' willingness to engage. Higher vaccine uptake was associated to a perception of the Covid-19 being a severe disease and a fear of immunization. The perception of most of the respondents about the Covid-19 vaccines was positively able to take the vaccine [2], [12]. As it is noticed in this study [22] To mobilize offline intervention, we noticed the use of social media to be strong predictors of the assumption that vaccines are harmful. The substantive impact of international misinformation is to raise the median country's number of adverse vaccine tweets. A substantial relationship exists between Social media organizations and general concerns about vaccines. Security. There is also a substantial relationship, Between campaigns of international disinformation and declining media attention for vaccines.

On the other side, this study discovered that unfavorable views regarding vaccines in general, such as potential side effects and long-term harm, did not play a small part in people's hesitation to get immunizations. Attempts to resolve numerous issues, particularly vaccination formulation, evaluation of vaccinations, and community engagement efforts, are expected to improve vaccination use [14]. Based on the main point of view distrustful attitudes were evident towards vaccination by ethnic minorities. The most likely reason for the lack of consensus in vaccination is low-income people and those who did not get a flu shot last year. Female gender, in living with children, and low income were factors contributing to the deaths of these mothers. For this study, an interpretation of the public's view on a proposed novel vaccine will be explored [19], [21]. As it is supported by this study [23] A significant proportion of participants in the Americas do not wish to be vaccinated if a COVID19 vaccine becomes available. For blacks, women, and conservatives, the probability of rejection is greater. Concerns over vaccine safety and efficacy are the two most mentioned reasons for rejection. Owing to concerns about protection and effectiveness, Blacks were much more likely to be hesitant than Whites.

Hesitancy and risk of taking the COVID-19 vaccine are linked to whether someone has already been 
vaccinated for influenza. More than half of the hesitant individuals expressed fear about potential adverse effects, according to the most frequent reasons provided for hesitancy [1], [8]. The current results can enable public health officials to approach vaccine-hesitant and resistant individuals more effectively, establish appropriate communication techniques that take into account their unique psychological arrangements and exploit networks of distribution that can reach these individuals successfully, According to the research, the UK and Ireland had lower acceptance rates and greater rates of hesitancy. When we compare our findings to those of similar research conducted in the United States and Ireland, we find lower rates [16]. There is also a noticeable finding of this study which supports by [24]. As this analysis gives valuable early estimates of Vaccination intentions and predictors of vaccination hesitancy; moreover, continued testing is carried out. If the COVID-19 situation progresses and a possible vaccine is developed, it is important.

With the outbreak of COVID-19, conspiracy theories emerged. The study examined the relationship between conspiracy beliefs, vaccine attitudes, and intention to be vaccinated. Men who were more likely to reject the vaccine than women were often considered useless because of the harmless presence of the dominant classes. The study found that conspiracy beliefs about chloroquine negatively predicted participants' intentions [4], [5]. On the other side, In the sample population, the majority were worried about the possible adverse effects of COVID vaccines. A person's desire to obtain a vaccine depends on their understanding that the vaccine has a beneficial, favorable effect. We should communicate the prosocial benefits and dangers of vaccination to educate the public [13], [17], [18].

Most health care workers believed it was significant. Half of the health care staff assumed that vaccines developed domestically would be safer than those produced overseas [7]. While more than half of survey participants decided to receive the vaccine as soon as it became available. Most people accepted that both the immunization plans and both forms of vaccines were safe [6]. It is also important to consider individuals who were distrustful of vaccination appeared to come from minority communities. Higher levels of low-income groups and those who have not received a flu vaccine last year correspond with lower vaccine confusion and rejection [20].

A majority of the research population decided that they would consent to receive vaccines if they have been proved safe and reliable. Fewer than half of the sample decided to get vaccinated even though it was offered free of charge [11], [15]. From the same point of view, the gender of participants was the only demographic factor affecting the intention to take the vaccine. Acceptance of the COVID-19 vaccine is high. high-risk populations.
Media strategies need to use social media and knowledge about health, emotional, social, and environmental effects [9], [10].

The government could expend a lot of resources in spreading consciousness of the value of vaccines among the risky community in Uganda. A large number of Jordanians are optimistic about their role in COVID-19 vaccine clinical trials. Among the determinants of such willingness are educational degree and past involvement in clinical trials as is supported by these findings [25][27].

\section{CONCLUSION}

acceptance of Covid-19 vaccines has a higher percentage among the Australian population. While the united states, the united kingdom, china, and Netherland shows a positive perception regarding the Covid-19 vaccine. They were overwhelmed to recognize the vaccine by the conspiracy belief and theory among the citizens of Italy. Overall the study found that majorities of the population from different countries have a different point of view regarding acceptance, hesitancy, and attitude as it demonstrated that majorities of them are hesitant regarding Covid-19 vaccine side effects and safety.

\section{ACKNOWLEDGMENT}

We would appreciate Hawler Medical University/ College of the nursing scientific committee.

\section{REFERENCES}

[1] J. Murphy et al., "Preparing for a COVID-19 vaccine: Identifying and psychologically profiling those who are vaccine hesitant or resistant in two general population samples," 2020.

[2] K. Pogue et al., "Influences on Attitudes Regarding Potential COVID-19 Vaccination in the United States," Vaccines, vol. 8, no. 4, Art. no. 4, Dec. 2020, doi: 10.3390/vaccines8040582.

[3] L. Shmueli, "Predicting intention to receive COVID19 vaccine among the general population using the Health Belief Model and the Theory of Planned Behavior Model," medRxiv, 2020.

[4] P. Bertin, K. Nera, and S. Delouvée, "Conspiracy Beliefs, Rejection of Vaccination, and Support for hydroxychloroquine: A Conceptual ReplicationExtension in the COVID-19 Pandemic Context," Front. Psychol., vol. 11, no. 10, pp. 1-9, 2020, doi: 10.3389/fpsyg.2020.565128.

[5] J. K. Ward et al., "The French public's attitudes to a future COVID-19 vaccine: The politicization of a public health issue," Soc. Sci. Med., vol. 265, p. 
113414, Nov. 2020, doi: 10.1016/j.socscimed.2020.113414.

[6] J. Wang et al., "Acceptance of COVID-19 Vaccination during the COVID-19 Pandemic in China," Vaccines, vol. 8, no. 3, Art. no. 3, Sep. 2020, doi: 10.3390/vaccines8030482.

[7] C. Fu, Z. wei, S. Pei, S. Li, X. Sun, and P. Liu, "Acceptance and preference for COVID-19 vaccination in health-care workers (HCWs)," Epidemiology, preprint, Apr. 2020. doi: 10.1101/2020.04.09.20060103.

[8] V. Grech, C. Gauci, and S. Agius, "Vaccine hesitancy among Maltese healthcare workers toward influenza and novel COVID-19 vaccination," Early Hum. Dev., p. 105213, Oct. 2020, doi: 10.1016/j.earlhumdev.2020.105213.

[9] L. P. Wong, H. Alias, P.-F. Wong, H. Y. Lee, and S. AbuBakar, "The use of the health belief model to assess predictors of intent to receive the COVID-19 vaccine and willingness to pay," Hum. Vaccines Immunother., vol. 16, no. 9, pp. 2204-2214, Sep. 2020, doi: 10.1080/21645515.2020.1790279.

[10] L. Williams et al., " Towards intervention development to increase the uptake of COVID - 19 vaccination among those at high risk: Outlining evidence - based and theoretically informed future intervention content," Br. J. Health Psychol., vol. 25, no. 4, pp. 1039-1054, Nov. 2020, doi: 10.1111/bjhp. 12468 .

[11] G. Graffigna, L. Palamenghi, S. Boccia, and S. Barello, 'Relationship between Citizens' Health Engagement and Intention to Take the COVID-19 Vaccine in Italy: A Mediation Analysis," Vaccines, vol. 8, no. 4, Art. no. 4, Dec. 2020, doi: 10.3390/vaccines8040576.

[12] S. S. Enitan, A. O. Oyekale, R. Y. Akele, K. A. Olawuyi, E. O. Olabisi, and A. J. Nwankiti, "Assessment of Knowledge, Perception and Readiness of Nigerians to participate in the COVID19 Vaccine Trial," Int. J. Vaccines Immun., vol. 4, no. 1, pp. 1-13, 2020, [Online]. Available: https://www.sciforschenonline.org/journals/vaccines /IJVI123.php

[13] D. Freeman et al., "COVID-19 Vaccine Hesitancy in the UK: The Oxford Coronavirus Explanations, Attitudes, and Narratives Survey (OCEANS) II," Psychol. Med., pp. 1-34, Dec. 2020, doi: $10.1017 /$ S0033291720005188.

[14] S. Qiao, C. C. Tam, and X. Li, "Risk exposures, risk perceptions, negative attitudes toward general vaccination, and COVID-19 vaccine acceptance among college students in South Carolina,"
medRxiv, p. 2020.11.26.20239483, Nov. 2020, doi: 10.1101/2020.11.26.20239483.

[15] R. M. Magadmi and F. O. Kamel, "Beliefs and Barriers Associated with COVID-19 Vaccination Among the General Population in Saudi Arabia," pp. 1-17, 2020, doi: https://doi.org/10.21203/rs.3.rs48955/v1.

[16] T. C. WITTE, "Preparing for a COVID-19 vaccine," PhD Thesis, Erasmus University, 2020. [Online]. Available: https://scholar.google.com/scholar?hl=en\&as_sdt=0 $\% 2 \mathrm{C} 5 \& \mathrm{q}=$ Preparing + for $+\mathrm{a}+\mathrm{COVID}-$ $19+$ vaccine+WITTE $\&$ btnG $=$

[17] S. R. Hursh, J. C. Strickland, L. P. Schwartz, and D. D. Reed, "Quantifying the Impact of Public Perceptions on Vaccine Acceptance Using Behavioral Economics," Front. Public Health, vol. 8, p. 608852, Dec. 2020, doi: 10.3389/fpubh.2020.608852.

[18] S. Khan, C. K. F. Rahman, H. C V, B. Jose, R. Tiwari, and K. Dhama, "COVID-19 Vaccine Acceptance: Beliefs and Barriers Associated with Vaccination Among the General Population in India," J. Exp. Biol. Agric. Sci., vol. 8, pp. S210-S218, Nov. 2020, doi: $\quad 10.18006 / 2020.8$ (Spl-1-SARS-CoV2).S210.S218.

[19] Y. A. Adebisi, A. J. Alaran, O. A. Bolarinwa, W. Akande-Sholabi, and D. E. Lucero-Prisno, "When it is available, will we take it? Public perception of hypothetical COVID-19 vaccine in Nigeria," medRxiv, vol. 1, no. 1, p. 2020.09.24.20200436, Oct. 2020, doi: 10.1101/2020.09.24.20200436.

[20] E. Paul, A. Steptoe, and D. Fancourt, "Attitudes towards vaccines and intention to vaccinate against COVID-19: Implications for public health communications," Lancet Reg. Health - Eur., p. 100012, Dec. 2020, doi: 10.1016/j.lanepe.2020.100012.

[21] H. Seale et al., "Examining Australian public perceptions and behaviors towards a future COVID19 vaccine," medRxiv, pp. 1-34, Sep. 2020, doi: 10.1101/2020.09.29.20204396

[22] S. L. Wilson and C. Wiysonge, "Social media and vaccine hesitancy," BMJ Glob. Health, vol. 5, no. 10, p. e004206, Oct. 2020, doi: 10.1136/bmjgh-2020004206

[23] T. Callaghan et al., "Correlates and Disparities of COVID-19 Vaccine Hesitancy," Social Science Research Network, Rochester, NY, SSRN Scholarly Paper ID 3667971, Aug. 2020. doi: 10.2139/ssrn.3667971.

[24] S. Butter, E. McGlinchey, E. Berry, and C. Armour, "Psychological, social, and situational factors 
associated with COVID-19 vaccination intentions: A study of UK key workers and non-key workers." PsyArXiv, pp. 1-26, Jul. 24, 2020. doi: 10.31234/osf.io/cfp3r.

[25] R. K. Abu-Farha, K. H. Alzoubi, and O. F. Khabour, "Public Willingness to Participate in COVID-19 Vaccine Clinical Trials: A Study from Jordan," Patient Prefer. Adherence, vol. 14, pp. 2451-2458, Dec. 2020, doi: 10.2147/PPA.S284385.

[26] I. Echoru, P. D. Ajambo, and E. M. Bukenya, "Acceptance and Risk Perception of COVID-19 Vaccine in Uganda: A Cross Sectional Study in Western Uganda," 2020.

[27] C. Laine, D. Cotton, and D. V. Moyer, “COVID-19 Vaccine: Promoting Vaccine Acceptance," Ann. Intern. Med., pp. 1-2, Dec. 2020, doi: 10.7326/M208008. 